\title{
Psicoterapia de la crisis
}

RESUMEN: La crisis es un sindrome agudo de estrés, caracterizado por là ruptura brusca de la vivencia de continuidad psíquica y por la respuesta activa ante un proceso de cambio. El éxito en esta respuesta favorece el desarrollo e integración de la personalidad, mientras que su fracaso puede llevar a una total desestructuración. La intervención terapéutica requiere consideración del tipo y fase de la crisis, de la personalidad y experiencias criticas del sujeto, y de los condicionantes socioculturales del entorno.

PALABRAS CLAVE: crisis, psicoterapia, estrés.

\begin{abstract}
Crisis is an acute stress syndrome, characterized by sudden break of the psychic continuity experience and by active response to a change process. Succes in this response favors personality development and integration, whereas failure carries the risk of total desestructuration. Therapeutic intervention has to consider the type and phase of the crisis, the personality and crisis experience of the subject, and the sociocultural conditions of the environment.

KEY WORDS: crisis, psychotherapy, stress
\end{abstract}

\begin{abstract}
"Hay un flujo y reflujo en los asuntos de los hombres, que, si se toma en la subida, lleva a la fortuna, y, si se descuida, toda la travesia de la vida queda encallada en bajios y en miserias"

Shakespeare, en Julio Cesar.
\end{abstract}

\section{El concepto de crisis}

Es frecuente asociar la idea de crisis con dificultad, riesgo y peligro. Sin embargo, la esencia del concepto está más próxima a la de cambio crucial, significativo o determinante. Procede de la raiz sánscrita skibh- cortar, separar, distinguir (1), asimilada por la voz griega krisis, decidir. El término fue usado por Hipócrates para referirse al momento en el en el que una enfermedad cambia su curso, para bien o para mal. Corominas recoge esta rancia raigambre médica al definir la crisis como "mutación grave que sobreviene en una enfermedad, para mejoría o para empeoramiento", $y$ añade, como segunda acepción más amplia, "momento decisivo en un asunto de importancia" (2).

La observación diacrónica de numerosos procesos naturales muestra interrupciones en su continuidad, a partir de las cuales, si el proceso continúa, lo hace de manera diferente a como lo habia hecho hasta entonces. Este punto de inflexión se corresponde tan bien con el concepto hipocrático de crisis, que su 
descripción puede servimos como definición. Así, entenderemos como crisis una condición inestable, que se presenta en el curso de un proceso, y cuya resolución condiciona y modula la continuidad del mismo. La crisis implica la inevitabilidad de una transformación. En sí misma, es una organización inestable y transitoria; de ahi el matiz perentorio, incluso angustioso, de su concepto. La incertidumbre y diversidad de posibilidades añade, por otra parte, la sensación de responsabilidad y de riesgo: En efecto, la crisis puede marcar el empeoramiento o la destrucción del proceso, pero también su fortalecimiento y optimización. Como dice Shakespeare por boca de Bruto, el grado de acierto de la acción en momentos de crisis marca la diferencia entre la miseria y la fortuna.

La aplicación del concepto de crisis en salud mental presupone un principio de continuidad psíquica, entendido como una experiencia básica subyacente a todas las demás. La interrupción de esta vivencia de continuidad es intolerable, y, si ocurre, debe ser rápidamente reparada. Es evidente que los seres humanos tienden a buscar una lógica interna en sus vidas, de tal manera que puedan entender su desarrollo y prever su curso. El sentido de la vida viene dado por esta lógica interna. La situación de crisis, al romper el flujo continuo de la existencia, fuerza un replanteamiento vital, obligando, a veces, a la construcción de un nuevo sentido. En la situación óptima, la aposición progresiva de vivencias personales, críticas o no, asegura la continuidad psíquica, la unificación de la consciencia y la constitución de una identidad autodefinitoria. Desde esta perspectiva, podemos entender la crisis como: una experiencia puntual que fuerza al individuo a reconsiderar en un plazo breve de tiempo el sentido de su vida. De ahí el riesgo y la potencialidad creativa de las crisis.

Cuando el proceso de resolución de crisis fracasa, la conciencia se fragmenta, las contradicciones internas se acumulan y el sentido personal de identidad se vuelve frágil y provisional. Como primer intento de compensación, las circunstancias y condiciones que amenazan la vivencia de continuidad son rechazadas, negando todo cambio y dificultad. Si esta defensa fracasa, la clara experiencia de la vida sin sentido conduce a la desesperación, y, para evitarlo, puede producirse como segunda línea de defensa la provocación o búsqueda de situaciones de cambio, con consecuencias a veces desastrosas.

Caplan (3), creador de la psiquiatría comunitaria, propone definir la crisis como "un obstáculo que el sujeto encuentra en la consecución de metas importantes y que no puede ser superado mediante sus estrategias habituales de resolución de problemas". El papel patogénico de las crisis viene de esta vivencia de incapacidad, de la sensación de ser superado por la situación, que puede acabar en una renuncia masiva al proyecto vital, con pérdida del futuro y desorganización de las capacidades de adaptación y defensa. De ahi que la intervención en la crisis se considere como una herramienta preventiva elemental en psiquiatría comunitaria (4). 
Naturalmente, las consideraciones negativas sobre la patogenicidad de las crisis no deben hacernos olvidar sus potenciales efectos beneficiosos. Toda crisis es un riesgo, pero también una oportunidad. La intervención del psiquiatra y del profesional de la salud mental debe buscar, no sólo la prevención de mayores males, sino también la optimización de los recursos personales (5). De ahí la importancia de los programas de formación profesional y de sensibilización y educación de la población en general. Sin ellos, los dispositivos sanitarios atenderán las situaciones de crisis con baja eficacia, e incluso con un posible grado de iatrogenia.

\section{Estrés, Trauma y Cambio.}

Desde que Cannon y Selye definieron, cada uno a su manera, el estrés fisiológico, el concepto ha sido extrapolado a las ciencias humanas, donde se le han atribuido significados diversos, como los de sobrecarga, exigencia exagerada, respuesta normal a situaciones anormales e, incluso, contrapartida fisiológica de la ansiedad. Desde el punto de vista psicosocial, podemos distinguir tres aspectos o grupos de factores que intervienen en esa peculiar interacción entre un individuo y su medio que denominamos estrés: a) Factores externos de estrés, que comprenden las circunstancias y acontecimientos del entorno que exigen un esfuerzo inhabitual del organismo para adaptarse a ellos. b) Factores internos de estrés, de los que depende la respuesta automática del organismo ante esas exigencias externas, y c) Factores moduladores, variables del individuo, como el estado afectivo, o del medio, como el apoyo social, que no están directamente relacionadas con la respuesta de estrés, pero que modifican la interacción entre factores externos e internos (6).

La morbilidad relacionada con el estrés depende de la intensidad y duración de los factores externos, de la reactividad individual y vulnerabilidad interna, y de los condicionantes ("moduladores") que inhiben o fortalecen los mecanismos de adaptación. Básico al concepto de estrés es el principio de homeostásis, o equilibrio interno del organismo, que se mantiene constante frente a todas las variaciones del entorno. Cuando la influencia del ambiente supera o no alcanza las cotas en las que el organismo responde con máxima eficiencia, éste percibe la situación como peligrosa o desagradable, desencadenándose una reacción de lucha-huida y/o una reacción de estrés, con hipersecreción de catecolaminas y cortisol (Ley General de la Homeostásis) (7)

Aunque los conceptos de estrés y trauma suelen estar asociados, corresponden en realidad a fenómenos distintos. El estrés implica siempre una dinámica de resistencia, tensión y lucha, que persiste mientras los factores externos lo exigen, o mientras dura la resistencia del organismo. El trauma indica ya una ruptura, un fracaso de las capacidades de integración, una discontinuidad en la vida psíquica. Lo que se llama estrés traumático no consiste en una mera sobrecarga temporal de las capacidades de adaptación, sino en una alteración profunda y permanente del funcionamiento mental. Por eso, las situaciones de estrés se recuerdan muy bien, mientras que los traumas se acompañan siempre 
de amnesia más o menos parcial. Para que un estrés se convierta en traumático, es necesario que su intensidad o su naturaleza superen las capacidades de procesamiento del sistema nervioso, dando lugar a disrupciones permanentes en la organización del mundo interno (8).

El cambio es parte fundamental de la existencia, verdad que constituye un elemento esencial de la antigua filosofía china. "El hombre sabio está siempre atento a la impermanencia de todas las cosas", dice Confucio en sus comentarios al I Ching, adelantándose 25 siglos a las modernas técnicas de inoculación de estrés. La investigación psicofisiológica muestra que la introducción de cambios o nuevas variables en una actividad altera los parámetros neurovegetativos y neuroendocrinos, incluso cuando esos cambios facilitan la actividad (7). Desde la más remota antigüedad es conocida la influencia de grandes estreses y traumas, pero el efecto de pequeños cambios y acontecimientos normales no ha sido comprendido hasta los trabajos pioneros de Holmes y Rahe (9). Según estos autores, cada acontecimiento nuevo, por banal que sea, exige un reajuste de las funciones habituales de la vida cotidiana. Un acumulo de muchos pequeños acontecimientos, en corto tiempo, puede llegar a ser equivalente a una gran tragedia. Cada cambio en nuestras vidas puede ser medido en "unidades de cambio vital", y la suma de estas unidades refleja el estado de nuestros factores externos de estrés por el periodo de tiempo considerado.

En el contexto de los síndromes de estrés, puede bien decirse que una crisis es una experiencia estresante, que en algunos casos llega a ser traumática, y que casi siempre provoca, o viene precipitada por, un cambio vital. El elemento distintivo de la crisis es la convicción o decisión interna de que la situación es insostenible y que es preciso hacer algo para transformarla. La motivación hacia el cambio es una respuesta apropiada a la percepción subjetiva de la naturaleza del estado de crisis.

\section{La evolución de una crisis.}

El desarrollo de una crisis sigue un proceso fásico, que se completa, habitualmente, en un tiempo inferior a ocho semanas. Siguiendo a Caplan (10) podemos diferenciar las siguientes fases :

Primera fase: Shock o Impacto agudo. Algo ocurre, generalmente de manera súbita, o, por lo menos, eso le parece al sujeto, que se siente afectado profundamente, sin poder evitarlo ni superarlo de la manera habitual en que hace frente a otros problemas. Junto al estrés propio de la situación, se añaden pronto sentimientos de confusión e impotencia, desamparo y desvalimiento. Inicialmente, cada individuo reacciona según sus pautas características de reactividad al estrés, entre las que predominan la ansiedad, la irritabilidad y las manifestaciones psicosomáticas, aunque algunos raros individuos experimentan entusiasmo y aumento de energía (11). 
Segunda fase: Desorganización critica. Cuando todas las estrategias habituales de afrontamiento y resolución de problemas fallan, la tensión emocional aumenta, hasta niveles insoportables. En este estado, diversas actividades pueden ser emprendidas, no para mejorar las cosas o resolver la situación, sino, simplemente, para escapar de ella o para descargar la tensión acumulada. As; , no son infrecuentes en esta fase conductas psicopáticas o "fuera de carácter", diversos tipos y grados de abuso de tóxicos, conductas autodestructivas y episodios emocionales bruscos. Característicamente, el sujeto despliega también actividades de búsqueda de ayuda, que pueden ser apropiadas y bien programadas, o "gritos de socorro" ineficaces y atropellados. Según empieza a hacerse evidente la insuficiencia de los recursos personales, y su disparidad con las exigencias de la situación, cada vez se hacen más prominentes sentimientos depresivos de indefensión y fracaso. France (12), aplicando la teoría de Seligman, distingue distintos grados de indefensión en una crisis, según que este sentimiento se adopte desde un punto de vista personal o universal, permanente o transitorio, y global o especifico. En el peor de los casos, el estado de indefensión es personal, permanente y global, es decir, el individuo se considera incapaz de afrontar una situación que otro cualquiera podría resolver, no tiene ninguna esperanza ni perspectiva de recuperarse, y extrapola su convicción de incompetencia e ineficacia desde el evento concreto a todas las áreas de su vida. En el mejor de los casos, el individuo considera su estado de indefensión como inevitable y propio de la naturaleza humana, afectando la situación a cualquier individuo tanto o más que a él mismo (universal), está convencido de que pasará (transitorio) y lo mantiene estrictamente restringido al evento concreto, conservado sensación de eficacia en todas las demás áreas de su vida (especifico).

Tercera fase: Resolución. Entre las múltiples actividades que el sujeto despliega ante el impacto inmediato de la situación, alguna puede tener éxito, y la crisis se resuelve casi sin llegar a plantearse. Pero en el desarrollo típico de una crisis, los primeros intentos de contraataque suelen fallar, y el individuo continúa sintiendo una creciente presión interna por hallar una solución. La urgencia de la situación puede estimular su creatividad, llevándole a descubrir nuevas estrategias de afrontamiento; o bien, puede encontrar nuevas fuentes de ayuda, formar nuevas relaciones o trabajar con profesionales o consejeros varios. Esta fase es el núcleo central de la crisis, en la que se toma una decisión, que puede significar la salida a través de la creación de nuevos recursos personales y de un crecimiento interno apresurado, o, por el contrario, a través de la consolidación de mecanismos maladaptativos de evitación y retraimiento. Lo cierto es que, en un plazo de tiempo limitado, el sujeto llega a una formulación más o menos consciente de la situación y de si mismo con respecto a ella.

Cuarta fase: Retirada final. Si la crisis no se resuelve, bien o mal, en las fases anteriores, se llega a la fase final, que parece en cierta forma análoga al estadio de agotamiento de la reacción general de adaptación de Selye. La retirada puede ser total, como a través del suicidio, o parcial, que puede ser interna, como a través de una desorganización 
psicótica o una estructuración delirante, o externa, como en un cambio radical de entorno, relaciones, actividad y hasta de nombre. En cualquiera de estos casos, la crisis se termina, sea por la muerte, sea por la negación de si mismo que supone la psicosis, sea por la renuncia a todo lo vivido hasta el momento de la crisis y el inicio de una nueva vida. France (12) llama la atención sobre la diferente calidad de las tentativas de suicidio que tienen lugar en la fase dos, que representan llamadas de ayuda o intentos de solución, y las que tienen lugar en la fase cuatro, que buscan directamente la muerte como manera definitiva de resolver la crisis. En todo caso, es preciso tener en cuenta que las tentativas de suicidio son frecuentes en las situaciones de crisis, sobre todo en adolescentes (13).

\section{Clasificación clinica de las crisis.}

Clínicamente, podemos conceptualizar la crisis como una situación transitoria de estrés, cuya resolución requiere una decisión estructurante, y en la que el sujeto tiene clara conciencia de la disparidad entre el rendimiento habitual de sus recursos y las exigencias de la tarea critica (14). Las manifestaciones de este estado dependen de la personalidad del sujeto, de sus experiencias anteriores, incluyendo su historia critica y traumática, de su entorno socio-cultural, de la fase en que llegue a nuestra atención, y del tipo de situación que precipite la crisis.

\section{Personalidad del sujeto.}

Como es de suponer, los rasgos de personalidad colorean la forma de afrontar la crisis, estableciendo, en primer lugar, un filtro sobre qué acontecimientos serán considerados como críticos (es decir, bloqueantes de las metas importantes del sujeto) y cuáles no. Así, la aparición de arrugas en la piel puede precipitar una crisis en una mujer con rasgos histriónicos, y pasar desapercibida a otra persona que no considere la belleza física un elemento importante de su identidad. En segundo lugar, una vez inmerso en la situación de crisis, los rasgos de personalidad condicionan la preferencia por determinadas estrategias de afrontamiento y de resolución de problemas, tema que ha sido admirablemente tratado por MacKinnon (15), y que no será repetido aquí.

Los dos parámetros de personalidad que más nos interesan, tanto por su influencia patoplástica como por su condicionamiento de las intervenciones terapéuticas, son la madurez y la flexibilidad. Entendemos por madurez el nivel de estabilidad del autoconcepto e identidad psicosocial. La flexibilidad es el grado o facilidad con que pueden formarse nuevas estrategias y decisiones, renunciando a otras que ya no cumplen el propósito por el que fueron creadas.

Naturalmente, a mayor madurez de la personalidad, mejor capacidad resolutoria, menor duración de la crisis y mejor salida de la misma. Es posible que la mayoría de los pacientes que llegan a nuestra atención en la fase II o en la IV de una crisis presenten diversos grados de inmadurez, lo que es tanto como decir de vulnerabilidad a la crisis. 
Por eso, las mejores medidas preventivas ante las crisis son aquellas que conducen al fortalecimiento y desarrollo de la personalidad, es decir, una buena educación. Sin embargo, desde el punto de vista terapéutico práctico inmediato, la intervención en la crisis no puede aspirar a influir de manera estructurante en la personalidad, debiendo conformarse con apoyar la frágil identidad comprometida, identificar pronto el área en que debe concentrarse el trabajo psicoterapéutico, y confiar en que las capacidades creativas del paciente se avivarán ante la necesidad del momento.

El repetido argumento sobre el valor curativo de las crisis reposa en que, si todo sale bien, no sólo ésta será superada, sino que el sujeto avanzará en su desarrollo personal, mejorando rasgos de vulnerabilidad o tendencias francamente patológicas. Por eso, desde el punto de vista de la teoría de la crisis, la personalidad se considera en un equilibrio dinámico, estructurado en base a decisiones y pautas de adaptación formadas en experiencias previas, pero predispuesto a la reorganización, ampliación y transformación, según sea necesario para responder a nuevas crisis. La flexibilidad de la personalidad viene dada por el grado en que el sujeto está abierto a nuevas experiencias y por su disposición al cambio interno. Aunque existe una cierta tensión entre madurez y flexibilidad, no son principios contradictorias, sino complementarios: Puede decirse que el desarrollo óptimo y armónico de la personalidad lleva a una madurez flexible y a una flexibilidad madura.

\section{Experiencias previas.}

Cada nueva crisis actúa no solamente por si misma, sino además como un recordatorio de crisis previas. En la medida en que éstas hayan sido mal resueltas, su reactivación será un estrés sobreañadido a la situación critica actual. Por el contrario, en la medida en que la resolución de crisis anteriores haya sido eficaz, el sujeto enfrentará la nueva con mayor confianza. Una historia de fracasos repetidos en la resolución de crisis es de mal pronóstico para la crisis actual, a menos que se consiga poner estos fracasos en perspectiva, elaborar las dificultades y bloqueos que impidieron resoluciones satisfactorias en el pasado, e incluir entre las decisiones a formar durante la nueva crisis la de "que no vuelva a pasar lo mismo". Es por eso importante, en la evaluación de un paciente en situación de crisis, obtener una buena historia crítica, especialmente en lo que se refiere a su actitud ante situaciones similares anteriores. Las pautas de enfrentamiento y las consecuencias experimentadas anteriormente nos dan una clara predicción de las probabilidades en la situación presente, y nos orientan hacia las avenidas terapéuticas más eficaces.

A pesar de que, en teoría, una crisis se resuelve en un plazo determinado, generalmente estimado entre dos y ocho semanas, hay muchos casos en los que, si las circunstancias exteriores lo permiten, la crisis queda fuera de la consciencia y de las actividades inmediatas, encapsulada, pero activa y pendiente de resolución. Cuando una nueva situación evoca una crisis pendiente, la reacción conjunta puede parecer 
catastróficamente exagerada. El terapeuta bien informado debe ser consciente de que está asistiendo a la resolución, no sólo de la crisis actual, sino, sobre todo, de la crisis fantasma o "asignatura pendiente". De hecho, acontecimientos aparentemente banales pueden desencadenar una crisis porque sirven de gatillo disparador de un problema largamente larvado, que ya no puede mantenerse por más tiempo fuera de la consideración consciente inmediata.

\section{Condicionantes socioculturales}

La educación, la cultura, y consideraciones similares, influyen, en primer lugar, en que una situación se considere como critica, o no. La pérdida del trabajo del cabeza de familia, por ejemplo, es una grave crisis en la mayoría de los casos, pero algunas comunidades rurales, con fuertes y amplios lazos familiares, y acceso a otros medios de subsistencia pueden afrontar la exigencia mejor que, por ejemplo, una familia obrera urbana. El apoyo social, en todos sus aspectos, es un importante modificador de la crisis, amortiguando el estrés e incrementando los recursos de afrontamiento, tanto por aporte directo como indirectamente por fortalecimiento auxiliar de la personalidad del sujeto.

Las pautas culturales de afrontamiento y resolución de crisis, compartidas por la comunidad y avaladas por una larga tradición, son sumamente útiles, y representan un sistema organizado de intervención y prevención en muchas crisis previsibles y normativas. No es lo mismo perder a un ser querido en un lugar que conserva sinceramente los rituales de duelo y atención a los moribundos, que en una moderna factoría de salud. Las pautas culturales establecidas guían a la persona que se enfrenta a la crisis en cuanto a su correcta conducta y actitud, favorecen la adecuada expresión emocional, aseguran el apoyo de familiares, amigos y extraños, garantizan la transmisión de los bienes y responsabilidades del muerto sin culpa, y, sobre todo, reconocen que se trata de una crisis personal importante, a la que hay que dedicar tiempo y sufrimiento. La moderna (o postmoderna) tecnología de la muerte y el duelo no permite ninguna de estas cosas, y es responsable de la masiva negación de aspectos esenciales de la vida, con graves consecuencias en cuanto a la salud mental de la población.

El afrontamiento y resolución de crisis también se puede aprender y compartir. La familia, como organización protectora responsable inmediata de la educación del individuo, juega en ello un papel principal. Como en tantas otras cosas, es más el ejemplo viviente que la normativa verbal lo que cuenta: Las crisis ajenas cuya resolución el niño tiene oportunidad de presenciar, son mucho más estructurantes que todos los consejos preventivos que pueda recibir.

Una familia funcionante tiene capacidad para repartir entre sus miembros el peso de una crisis, tanto si es global como si afecta principalmente a uno de ellos. Entre las posibilidades más frecuentes se incluyen: a) la distribución de papeles ante la crisis 
a afrontar, b) la potenciación colectiva de sus recursos individuales, c) el descargo del rol familiar de la persona más afectada en otro miembro, d) el apoyo emocional desculpabilizante y creativo.

Una familia disfuncional puede fácilmente pervertir los mecanismos de la familia sana, por ejemplo a) descargando el conjunto de sus problemas individuales en un chivo expiatorio, que se convierte así en el depositario de las insuficiencias familiares; b) ventilando las tensiones derivadas de la crisis en agresiones internas y reproches culpabilizantes; c) exigiendo despiadadamente al miembro en crisis el cumplimiento de sus responsabilidades, familiares u otras, o, por el contrario, d) eximiéndole de manera extrema y permanente de las mismas, lo cual acaba siendo una forma de descalificación más que de ayuda.

El entorno académico o laboral también juega un papel condicionante, a favor o en contra, como depositario de pautas de apoyo o de persecución en tiempos de crisis. El crecientemente reconocido fenómeno de acoso psicológico institucional (16) merece ser tenido en cuenta, no tanto por su importante participación en la génesis de estados críticos, sino, sobre todo, por el efecto inhibidor que tiene sobre las capacidades de afrontamiento y resolución del sujeto. El terapeuta avisado debe explorar esta posibilidad, y no asumir que toda victimización está sólo en la mente del paciente o ha sido causada por él mismo. La posibilidad de verbalizar, compartir, comprender y reaccionar ante una experiencia de acoso es vital para recuperar la moral y confianza ante una situación de crisis.

\section{Tipos de crisis.}

Aunque la teoría general se aplica a todos los tipos de crisis, es importante para una intervención apropiada tener en cuenta las circunstancias concretas en que pueden surgir, tanto en cuanto a su contenido como en cuanto a sus aspectos formales. Con respecto a estos últimos, es útil distinguir entre: a) Crisis generalizadas, que afectan a todo un grupo o familia, y personales, que afectan sólo a un individuo concreto; b) Crisis inesperadas e imprevisibles, como catástrofes, accidentes de tráfico, etc., en las que no es posible una preparación previa, y anticipadas o previsibles, como la muerte de un familiar mayor, un divorcio largamente anunciado, etc.; c) Crisis normativas, un tipo especial de crisis previsible, relacionado con la emergencia de funciones y necesidades nuevas en edades criticas; y d) transiciones o cambios vitales importantes no incluibles en los apartados anteriores, cuya característica principal es que son, en gran medida, conscientemente autogenerados.

Las crisis generalizadas son las que afectan a todo un grupo, comunidad $u$ organización, y sus causas tienden a ser relativamente objetivas y externas. Son éstas las circunstancias que ponen a prueba la calidad del liderazgo establecido, y permiten la emergencia del héroe, entendido como el individuo que toma sobre si la carga y 
los problemas de la comunidad, y los resuelve. Por el contrario, las crisis personales o privadas afectan a una persona concreta, de manera relativamente independiente al acontecer sociocultural general. Aunque posiblemente interesantes para ella, los problemas a resolver no afectan de manera central a la comunidad a la que pertenece el individuo, por lo su respuesta es variable, pudiendo llegar a no responder en absoluto. El origen de las crisis personales, más que con las circunstancias, está relacionado con una particular combinación de carácter, oportunidad y destino. En efecto, algunas personas atraviesan repetidamente crisis personales que para otros son totalmente desconocidas, como si estuvieran ensayando soluciones en la vida real para aplicarlas a problemas que llevan en su interior.

Aunque conceptualmente hemos incluido aquí las crisis accidentales e imprevisibles, su estudio concreto detallado pertenece al campo del estrés traumático (8). Las crisis previsibles están relacionadas con propiedades de la vida humana, las estructuras sociales en que se desarrolla, los usos y costumbres de su tiempo y lugar, y el entorno cultural inmediato o dominante en que se integra.

Las crisis normativas, descritas inicialmente por Erik Erikson $(17,18)$, son universales, en el sentido de que ocurren en todos los seres humanos, y, al mismo tiempo, personales, en el sentido de que, para cada individuo, es una vivencia intransferible, consubstancial a su propio desarrollo psíquico. Cada ser humano nace con un conjunto general de aptitudes, capacidades y tendencias, que conforman una constelación genética cuya actualización está modulada por las influencias de la educación y del medio ambiente. La maduración de la personalidad requiere el dominio secuencial de una tarea psicosocial básica, especifica a cada etapa del desarrollo. Cada crisis se plantea como una oposición dinámica entre dos actitudes extremas que han de desarrollarse en el curso de la relación interpersonal clave de esa edad. Un extremo representa la actitud correspondiente con la resolución óptima de la tarea crítica y el otro, su fracaso total. Según van sucediéndose las crisis normativas a lo largo de toda la vida, su dominio progresivo asegura una creciente integración y riqueza de la personalidad. La superación de cada crisis normativa se acompaña de la adquisición de una virtud o cualidad humana básica, que Erikson define como "fuerza interna o cualidad activa', cuyo ejercicio da evidencia del grado de resolución de la tarea psicosocial correspondiente. Cada dinámica crítica debe entenderse como un continuo entre dos extremos, y no como una dicotomía exacta. Por esta razón, es difícil encontrar personas que hayan triunfado o fracasado totalmente en alguna etapa del desarrollo, siendo lo habitual haber alcanzado un cierto grado de maestría en casi todas ellas.

La razonable superación de cada tarea crítica a la edad apropiada, con la adquisición de la habilidad psíquica correspondiente, sitúa al individuo en condiciones óptimas de afrontamiento para la siguiente fase de su desarrollo psicosocial. Inversamente, cada fallo relativo en una tarea dificulta el éxito en las etapas subsequentes, y va 
marcando al sujeto con sentimientos negativos específicamente asociados con cada una de esas crisis.

En el siguiente cuadro sinóptico presentamos las etapas correspondientes a cada edad, junto con su crisis psicosocial característica, la persona relacionada con la tarea crítica, su correspondencia con la etapa de desarrollo psicosexual según la clasificación de Freud, y la cualidad moral que se adquiere con el éxito en su resolución.

\begin{tabular}{|c|c|c|c|c|}
\hline Edad & $\begin{array}{c}\text { Crisis } \\
\text { Psicosocial }\end{array}$ & $\begin{array}{l}\text { Persona } \\
\text { relacionada }\end{array}$ & $\begin{array}{c}\text { Fase } \\
\text { de Freud }\end{array}$ & $\begin{array}{c}\text { Cualidad } \\
\text { moral }\end{array}$ \\
\hline $0-2$ & $\begin{array}{l}\text { Confianza, } \\
\text { desconfianza. }\end{array}$ & madre & oral & Esperanza \\
\hline $1-3$ & $\begin{array}{l}\text { Autonomía, ver- } \\
\text { güenza y duda }\end{array}$ & padre & anal & Voluntad \\
\hline 3- 6 & Iniciativa, culpa & $\begin{array}{l}\text { núcleo } \\
\text { familiar }\end{array}$ & fálica & Decisión \\
\hline $5-13$ & $\begin{array}{c}\text { Industriosidad, } \\
\text { inferioridad }\end{array}$ & escuela & latencia & Habilidad \\
\hline $13-20$ & $\begin{array}{l}\text { Identidad, } \\
\text { difusión de la } \\
\text { identidad }\end{array}$ & $\begin{array}{c}\text { compañeros } \\
\text { (grupo de } \\
\text { iguales) }\end{array}$ & & Fidelidad \\
\hline $18-30$ & $\begin{array}{l}\text { Intimidad, } \\
\text { Aislamiento }\end{array}$ & cónyuge & genital & Amor \\
\hline $20-30$ & $\begin{array}{c}\text { Creatividad, } \\
\text { Ensimismamiento }\end{array}$ & $\begin{array}{l}\text { hijos y de- } \\
\text { pendientes }\end{array}$ & & Ternura \\
\hline 45- - & $\begin{array}{l}\text { Integridad, } \\
\text { desesperación }\end{array}$ & Humanidad & & Sabiduría \\
\hline
\end{tabular}

Fases de desarrollo psicosocial según ERIKSON, y su relación con las de desarrollo psicosexual de Freud y la formación de las cualidades humanas básicas. Cada crisis se plantea como una oposición dinámica entre dos puntos extremos, indicando el primero la resolución óptima de la tarea crítica, y el segundo su fracaso total (Rivera, 1979) 
Las transiciones, pasajes o cambios vitales importantes son crisis relativamente predecibles, relacionadas con el propio proceso de crecimiento / envejecimiento, y su ajuste en un entorno social altamente regulado. Desde un planteamiento diferente, Gail Sheehy (20) coincide con Erikson en la periódica necesidad de cambiar el marco de referencia que nos ha guiado durante una etapa de nuestra vida, para ajustarnos mejor a nuevas condiciones que, de manera bastante predecible, surgen y se establecen cada cierto tiempo. Algunos ejemplos son la constitución de una familia, el nacimiento de hijos, la adultez y emancipación de éstos, la jubilación, las enfermedades graves o crónicas, los cambios fisiológicos relacionados con el climaterio ... Desde este punto de vista, es sorprendente que los sistemas educativos no preparen mejor a la población para afrontar crisis que son, no sólo inevitables, sino, sobre todo, parte del propio proceso vital. Muy por el contrario, algunas corrientes socioculturales favorecen su negación, minando así la capacidad natural de adaptación y afrontamiento. Algunos ejemplos son el culto a formas de belleza poco naturales, el hedonismo, la huida del compromiso en favor de la conveniencia, la justificación de la injusticia y la violencia, la perversión de la democracia a través del clientelismo y el miedo...

La aceptación del cambio como parte natural de la existencia facilita enormemente su manejo, y por eso algunos expertos, como Bridges (21), recomiendan abiertamente programas de psicoeducación preventiva. Dentro de esta tendencia, el programa de Gestión Creativa del Cambio (Creative Change Management - CCM), estructurado en cuatro fases, enseña y desarrolla conceptos, habilidades y actitudes que facilitan el control y dirección del tránsito entre dos estados, con el objetivo, no sólo de que el segundo estado sea mejor que el primero, sino, sobre todo, de que la persona que atraviesa el proceso sea al terminarlo mejor de lo que era al comenzarlo (5). El primer paso es la renuncia al estado anterior, proceso equivalente al de duelo, porque no es posible empezar nada nuevo mientras se siga aferrado a lo viejo. El segundo es el reconocimiento de la inseguridad como un estado natural, en el que se puede aprender y en el que es posible, y hasta interesante, vivir. El tercero es el descubrimiento y estructuración de la nueva realidad. El cuarto es la integración de las nuevas estructuras en un proceso de continuidad de la vivencia, que permite aprovechar y dotar de sentido las experiencias pasadas. Este método va más allá de la mera adaptación o superación del estrés, potenciando las posibilidades de desarrollo personal inherentes a la situación crítica.

\section{Detección y Diagnóstico de las crisis.}

Las manifestaciones externas o aparentes de las crisis son variadas, múltiples y dificiles de describir en términos operativos. Con frecuencia, cumplen criterios de trastorno adaptativo o de trastorno posttraumático de estrés, pero no todos los cuadros con esos diagnósticos son crisis, ni todas las crisis llegan a ser diagnosticadas. Gran parte de lo que denominamos "morbilidad psiquiátrica menor", fenómeno muy extendido en la 
población, corresponde probablemente a situaciones de crisis sin detectar (22). Después de cierto tiempo, muchas de estas situaciones acaban por cumplir los criterios de alguna condición de corte depresivo, aunque también pueden cumplir los de algún trastorno de ansiedad o de adicción.

El hecho de que las crisis sean difíciles de diagnosticar como tales en sentido desemétrico estricto, junto con la importancia de su detección precoz, nos obliga a replantearnos la conceptualización del diagnóstico en psiquiatría. En otro lugar, he defendido el criterio de que la actividad diagnóstica ha de estar enfocada al beneficio del enfermo, esto es, la detección del origen de su sufrimiento debe facilitar su erradicación o al menos su alivio, así como la prevención de mayores males (23). Las actuales tendencias diagnósticas, 'ateóricas', operativas y universalizantes, no facilitan grandemente esta tarea. Para evitar confusiones, antes de proseguir, recordaremos algunos conceptos fundamentales (22):

El diagnóstico representa la decisión final del médico con respecto a la relevancia patológica de una determinada agrupación de signos, síntomas y conductas. Los criterios estandarizados aseguran una relativa constancia del diagnóstico a través de todo el mundo, particularmente en lo que se refiere a los trastornos más intensos y mejor definidos. El malestar subjetivo, por otra parte, es el sufrimiento, descontento o sensación desagradable que un individuo experimenta en un momento determinado, independientemente de cómo lo exprese. La queja principal surge en la ocasión de la consulta con un profesional de la salud, y representa una decisión del paciente sobre los motivos apropiados para solicitar asistencia clínica. Finalmente, la conducta de enfermedad es la manera personal en que el individuo interpreta su malestar subjetivo y reacciona frente a él. La validez de un sistema diagnóstico queda gravemente afectada si no tiene sensibilidad a los tres últimos factores, que a su vez están relacionados con la cultura, costumbres y actitudes del sujeto. El malestar subjetivo no siempre es fácil de describir en los términos operativos de la escuela de Saint Louis. La queja principal puede ser reflejo fiel del malestar subjetivo, o bien puede ocultarlo, deformarlo o tamizarlo para ajustarse mejor a normas culturales. La conducta de enfermedad no siempre incluye el recurso a profesionales cualificados, sobre todo si no se percibe a éstos como proclives a escuchar y entender todo lo que el enfermo necesita comunicar.

Por otra parte, para que un diagnóstico sea de beneficio al enfermo, es decir, cumpla sus aplicaciones éticas (23), es necesario que ofrezca indicaciones sobre los procesos subyacentes en el sufrimiento o disfunción del enfermo, tarea que Jimenez Diaz llamaba el "diagnóstico patogenético". En psiquiatría, esta forma de diagnóstico requiere la consideración continua de los factores psicosociales y psicodinámicos durante toda la entrevista. 


\section{La intervención en la crisis.}

Caplan y su escuela son los principales constructores de la teoría y la práctica de intervención en la crisis (3), pero el origen del concepto debe reconocérsela a Lindeman (24), que demostró la importancia del tratamiento intensivo breve en reacciones de duelo subsecuentes a crisis inesperadas generalizadas. La intervención en la crisis es un procedimiento especifico cuyo objetivo principal es modificar la relación de fuerzas en lucha que forman la crisis, incrementando la probabilidad de inclinar la balanza hacia el lado positivo. Una pequeña corrección de trayectoria en el momento oportuno tiene grandes efectos.

Las cualidades del psicoterapeuta son cruciales para este tipo de trabajo, por la extrema susceptibilidad del paciente a la calidad de la relación y su fácil influenciabilidad externa. No hay técnica que funcione con un terapeuta patoso o malévolo, e, inversamente, un buen terapeuta acierta de manera intuitiva con la acción apropiada, aún en las situaciones más complicadas. Por otra parte, gran parte del aprendizaje en el afrontamiento y resolución de crisis tiene lugar de manera incidental, esto es, por absorción progresiva de las actitudes del terapeuta con ocasión de algunas de sus intervenciones concretas.

\section{La formación personal del terapeuta.}

La formación en psiquiatría es inherentemente estresante, hasta el punto de que muchos residentes en esta especialidad muestran signos de estrés postraumático (25). Las causas de este sufrimiento psíquico no dependen sólo de las dificultades y exigencias del contacto interpersonal con el enfermo, sino también de factores internos que favorecieron, en primer lugar, la elección de la especialidad. Es frecuente que, a lo largo del periodo de residencia, tenga lugar una crisis, relacionada con la formación de la identidad profesional, $\mathrm{y}$, más profundamente con nuevos intentos de resolución de problemas adolescentes reactivados (26). En la salida óptima de esta crisis se forman los rasgos de madurez que constituyen las cualidades esenciales del psicoterapeuta: Empatia objetiva, Aceptación dinámica del paciente, Tolerancia de la angustia, Libertad de la consciencia, Ausencia de gratificación a expensas del paciente y Motivación creativa (27). Al menos en cierto grado, las cualidades psicoterapéuticas deben estar constitucionalmente presentes, su desarrollo óptimo se facilita con el tratamiento personal, y el dominio de su aplicación clinica es entrenable con la metodología docente adecuada.

\section{Establecimiento de la relación terapeútica.}

Más que en otros contextos, la relación en si misma es importante. La actitud empática, genuina y sincera del terapeuta es esencial, evitando muestras de compasión o conmiseración, y haciendo patente su interés por el paciente y su crisis, especialmente en sus elementos subjetivos. La aceptación del paciente en toda su dimensión, tanto en su sufrimiento como en sus capacidades de autorregulación, tanto en sus tendencias más creativas como en sus afectos más negativos y destructivos, favorece la comunicación y el desbloqueo. Más que con instrucciones directas, la correcta actitud del terapeuta 
trasmite de manera incidental una sensación de cooperación y trabajo, desculpabilizando y favoreciendo la recuperación progresiva de sentimientos de competencia y eficacia.

Escuchar es esencial, dejando al paciente la guia principal, pero introduciendo cuando necesario pequefias correcciones de trayectoria que faciliten la clarificación, la catarsis, el descubrimiento de los recursos personales y la exploración de nuevas posibilidades de solución. No se trata de contradecir, dar instrucciones o convencer, sino de abrir canales mentales hacia alternativas liberadoras del circulo vicioso en que tiende a encerrarse. No importa mucho acertar a la primera ni guiar hacia un terreno prefijado; el proceso de corrección de trayectoria es, en sí mismo, más importante que sus contenidos concretos. La experiencia de que las decisiones o consideraciones que uno va tomando no son forzosamente necesarias, y que siempre existen alternativas, va creando insensiblemente un fondo de esperanza, a la vez de desarrolla una habilidad imprescindible para la resolución de problemas.

\section{Atención a preservar relaciones importantes.}

Anexo al establecimiento de una buena relación terapéutica está el mantenimiento y mejora de las relaciones interpersonales clave. No sirve de mucho nuestro esfuerzo si el paciente debe enfrentarse después con actitudes disfuncionales como las descritas en el apartado de condicionantes socioculturales, o si la propia situación de crisis ha ido generando reacciones de rechazo, agresividad o distanciamiento en sus personas de apoyo. Idealmente, debe entrevistarse al cónyuge o personas importantes para evaluar su actitud e instruirles sobre la mejor actitud para con el paciente. No es infrecuente que esa persona clave esté también sufriendo los efectos de la crisis, y que pueda necesitar tratamiento, en ocasiones con más urgencia y rendimiento que el paciente designado. En caso de colaboración negativa, puede ser necesario clarificar con el paciente los sentimientos que sus relaciones principales generan en él, ayudarle a entender y protegerse de las reacciones disfuncionales, $y$, extremo último muy importante, prevenirle contra actitudes destructivas de relaciones importantes que él mismo pueda desarrollar bajo los efectos de la tensión generada por la crisis.

\section{Técnicas de gestión o resolución emocional}

Dar salida a respuestas emocionales contenidas es prioritario en el tratamiento de la crisis. La percepción y expresión de emociones relacionadas con la pérdida y el duelo son especialmente importantes. Algunos concomitantes fisiológicos, como el llanto, han sido con frecuencia bloqueados por la educación, y el paciente puede necesitar aclaraciones acerca de su importancia y utilidad. La ventilación emocional puede tener lugar de manera incidental desde la primera entrevista, o de manera deliberadamente estructurada a través de técnicas como el análisis autógeno o similares $(28,29,30)$. La actitud del terapeuta debe ser simultáneamente permisiva y contenedora, desdramatizando la experiencia disfórica, y educando sobre el significado y la metodología de la descarga 
emocional. Las emociones son tratadas como indicadores de estados internos y como la expresión de decisiones y juicios extraconscientes; se aceptan, por lo tanto, como fuentes de información, al mismo tiempo que se desactivan como sufrimientos indeseables.

\section{Recontextualización.}

Una crisis significa una ruptura con los puntos de vista habituales, y en ella se barajan elementos que no pueden ser integrados en el mundo interno sin crear notable destrucción del mismo. Recontextualizar es poner las cosas en perspectiva, redefinir las situaciones y decidir actuaciones en términos más acordes con una visión positiva de la realidad. No se trata de engañarse, edulcorar las cosas o minimizar las tragedias, sino de operar desde un punto de vista que permita sacar partido de la experiencia. Así, al dar prioridad a la información disponible para resolver la crisis, se evita concentrarse en la experiencia disfórica como principal contenido de la conciencia. Priorizar tiene efectos paradójicos, por cuanto que concentrarse en el problema y aplicarle técnicas de resolución y recontextualización es más ansiolítico que intentar pensar en otra cosa.

\section{Recuperación y entrenamiento de capacidades pro-homeostáticas.}

Nuestro organismo está dotado de mecanismos automáticos de autorregulación, encargados de mantener el equilibrio interno frente a los cambios y agresiones del entorno. Con frecuencia, la operación de estos procesos pro-homeostáticos se confunde con la causa del sufrimiento que se quiere evitar, y, en lugar de facilitar sus funciones, se intenta inhibirlas. Así, por ejemplo, cuando en medio de una crisis el sujeto afecto se despierta completamente despejado a las cuatro de la mañana, es posible que no se trate de un insomnio a combatir con hipnóticos, sino de una sabia artimaña cerebral para trabajar intensamente durante unas horas en un escrito vital, en la organización y revisión de documentos, etc, 0 , simplemente, para meditar sosegadamente sobre la situación y sus posibles soluciones. Inversamente, cuando en periodos de intenso cambio el sujeto se nota necesitado de más horas de sueño de lo habitual, puede que eso sea lo acertado, ya que dormir facilita la reelaboración de información contradictoria y la consolidación de nuevas memorias. Es frecuente observar que las técnicas de meditación inducen estados naturales que no solamente potencian la capacidad de resistir el estrés, sino que además facilitan la reconexión y reelaboración de procesos mentales, con la consiguiente reestructuración del mundo interno (31).

\section{Medicación psicotropa.}

La intervención en la crisis es fundamentalmente un procedimiento psicoterapéutico. Pero ello no obsta para que, en algunos casos, pueda ser conveniente asociar tratamiento psicofarmacológico, ilustrado por el diagnostico patogenético, e integrado con la estrategia terapéutica general. Los antidepresivos de nueva generación son útiles por su efecto incrementador de la resistencia al estrés, sobre todo cuando la sintomatología depresiva está ya presente. Cuando en el curso del afrontamiento de la crisis aparecen cuadros depresivos serios, es difícil obtener una buena respuesta a la psicoterapia antes 
de que se logre una cierta recuperación farmacológica de la función limbo-hipotalámica. Los ansiolíticos son menos útiles, y, en todo caso, de rendimiento inferior a un buen entrenamiento en relajación o meditación. Sin embargo, deben ser tenidos en cuenta en las disomnias de estrés, utilizándolos como ayuda provisional, no como solución definitiva. Naturalmente, cuando el paciente presenta síntomas que desbordan la respuesta de estrés relacionada con la crisis, como en las psicosis reactivas, el tratamiento farmacológico debe ser enérgico y apropiado. Es preciso, además, tener en cuenta que procesos psicóticos silentes y predisposiciones depresivas pueden ser reactivados en situaciones de crisis, estando indicado en este caso el tratamiento preventivo con estabilizadores del humor, como litio, carbamazepina o clonazepam. 


\section{BIBLIOGRAFÍA}

1. Roberts, EA y Pastor, B: Diccionario etimológico indoeuropeo de la lengua española. Alianza, Madrid, 1996

2. Corominas, J: Breve diccionario etimológico de la lengua castellana. Gredos, Madrid, 1961

3. Caplan, G: An approach to community mental health, Grune\&Stratton, New York, 1961

4. Caplan, G: Principles of preventive psychiatry. Basic Books, New York, 1964

5. Optimización de Recursos Personales. http://www.psicoter.es/ORP

6. Gonzalez de Rivera, JL: "Factores de estrés y enfermedad. Actas Luso-Esp. Psiquiatr. Neurol. 19: 290-297 (1991)

7. González de Rivera, JL: “Estrés, homeostasis y enfermedad". En: JLG de Rivera, A. Vela y J. Arana: Manual de Psiquiatria, Karpos, Madrid, 1980

8. Gonzalez de Rivera, JL : "El sindrome post-traumático de estrés (SEPT)". Psiquis, 11:290-298 (1990)

9. Holmes, TH y Rahe, RK: "The social readjustment rating scale". J. Psychosom. Res., 1967, 11:213-218

10. Caplan, G: Support systems and community mental health. Behavioural Publications, New York, 1974

11. Gonzalez de Rivera JL, De las Cuevas C, Monterrey AL, RodriguezPulido F, Gracia R: "Stress Reactivity in the General Population". Eur. J. Psychiatr., 1993, 7:5-11

12. France, K: Crisis intervention. A handbook for immediate person-to- person help. Charles C. Thomas, Springfield, 1982

13. Runeson, B: "Mental disorder in youth suicide. DSM-III axes I and II". Acta Psychiatr. Scand, 1989, 79:490-496

14. Gonzalez de Rivera, JL: "Nosologia psiquiatrica del estrés". Psiquis, 2001, 22 : 1-7

15. MacKinnon, RA y Michels, RM: The psychiatric interview in clinical practice. WB Saunders, Philadelphia, 1971

16. Gonzalez de Rivera, JL: "El acoso psicológico institucional". Diario Médico, 18 de Julio $2000: 14$

17. Erikson, EH: "Identity and the life cycle". Psychological Issues, 1959, 1 (1):1-171

18. Erikson, EH: Insight and responsability. WW Norton, New York, 1964

19. Gonzalez de Rivera, JL: "El estrés infantil". En: J. Arana (Ed.) Niños Dificiles. Karpos, Madrid, 1979

20. Sheehy, G: Passages. Predictable crisis of adult life. Bantam Books, New York, 1977

21. Bridges, W: Transitions: Making sense of life changes. Addison-Wesley, Reading, 1980

22. Gonzalez de Rivera, JL, Rodriguez-Pulido, F y A Sierra: El Método Epidemiológico en Salud Mental. Masson-Salvat, Barcelona, 1993

23. Gonzalez de Rivera, JL: "La ética del diagnóstico: aspectos clinicos". Psiquis, 17:263-278 (1996)

24. Lindemann, E: "Symptomatology and management of acute grief". Amer. J. Psychiatr., 1944, 101:141-148

25. Klamen, DL, Grossman, L, Kopacz, D, y Doblin, B: "Psychiatric residents show signs of post-traumatic stress". Psychiatric News, 1993, 28 (15): 7,20

26. Gonzalez de Rivera, J.L. "Identity and Psychiatric Training". J. Psychiatr Neurosci, 5: 24-27 (1980) 
27. Gonzalez de Rivera, J.L. "Psicoterapias y Psicoterapeutas". Psiquis, 3: 112-115 (1982)

28. Gonzalez de Rivera, JL: Psicoterapia Autógena. UNED, Madrid, 1999

29. Gonzalez de Rivera, JL “Autogenic Psychotherapy and Psychoanalysis”. En: J. Guimon (Ed.) The body in Psychotherapy. Karger, Basilea, 1997. págs. 176-181.

30. Gonzalez de Rivera, JL: "Autogenic Psychotherapy: The tool Freud was looking for". International Journal of Psychotherapy, 2001, 6:71-76

31. Gonzalez de Rivera, JL "Creatividad y Estados de Conciencia". Revista de Psicologia General y Aplicada, 33: 415-426 (1978)

Fecha de recepción: 28/5/01

* José Luis Gonzalez de Rivera y Revuelta

Catedrático de Psiquiatria

Instituto de Psicoterapia e Investigación Psicosomática

Avenida de Filipinas, 52 - 28003 Madrid 